\title{
Timetable synchronisation: Urban public transport in busy hubs of long-distance transport
}

\author{
Josef Bulíček ${ }^{1, *}$ \\ ${ }^{1}$ University of Pardubice, Faculty of Transport Engineering, Department of Transport Technology and \\ Control, Studentská 95, 53210 Pardubice, Czech Republic
}

\begin{abstract}
The article is focused on timetable synchronization problem. Interconnection of urban public transport and long-distance transport in busy hubs is researched. Intension is to find adequate time positions of urban public transport arrivals and departures with an effort to minimize passengers' time loss related to this interchange. Timetable of long distance transport is considered as constant (given). Timetable of urban public transport is result of optimization (and presupposed to be periodic). Nonlinear integer mathematical programming model is formulated. Model is implemented in Microsoft Excel Solver. Parallel way based on exhaustive-search algorithm is introduced as well. This algorithm provides extended set of output data able to be used in transport planning. Two different criterions are proposed. Each interchange (pair of vehicles) has the same weight in the case of uniform approach. Intensity approach considers interchanges as weighted by numbers of interchanging passengers. Some additional modifications of proposed model are mentioned for possibility to regard some important technological aspects and to reach more individualized solution. Model is illustrated by the case study located to Pardubice main station as a hub of passenger railway transport. Interface with urban public transport is mentioned as an illustrational example of synchronization.
\end{abstract}

\section{Introduction}

Introduction is divided into 3 parts: problem statement, literature overview and research hypothesis. The aim of this chapter is to create a background for proposal of a mathematical optimization model representing the core of the article. Model takes a part in habilitation thesis elaborated by the author of this article [1].

\subsection{Problem Statement}

The problem is how to synchronize timetables of urban public transport (UPT) in busy hubs of long-distance transport (LDT) like railway stations, bus terminals or airports. In detail,

\footnotetext{
*Corresponding author: josef.bulicek@upce.cz
} 
how to find adequate time positions of arrivals and departures for each UPT line operated in the hub. Directions of drive are considered separately on each UPT line in the article; each direction as individual line. Following 4 presumptions concretizing the problem are considered:

a) There is a relative large number of LDT services arriving and departing all over the time. The consequence is that it is not possible to make interconnections between all LDT and UPT services individually. Periodic timetable in LDT is possible, but not necessary - any set (marked as $J$ ) of individual LDT services can be taken into consideration.

b) Time positions of departures and arrivals in LDT are strictly given and not able to be changed (moved or modified). It is caused by priority of LDT with an effort to respect all conditions occurred in this prior network (e.g. synchronization of timetables in other important LDT hubs etc.).

c) Set of $I$ is consisted of all UPT lines operated in the hub. All these UPT lines are operated in periodic form. Individual services of UPT line $i \in I$ are going in strictly defined line headway (interval) of $L H_{i}$ (after $L H_{i}$ minutes).

d) All (or the majority) of lines in both subsystems (UPT and LDT) are routed through the hub. The hub is a stop on their route only. Dwell (sojourn) times at hub $D T_{i}$ are limited, because $D T_{i}$ causes time loss for passengers continuing by the same service (vehicle) with no interchange. These dwell (sojourn) times $D T_{i}$ are usually shorter than necessary (minimal possible) transfer time $N T T_{i j}$ needed for walking between platforms for UPT line $i \in I$ and LDT service $j \in J$ (or $N T T_{j i}$ for interchange from LDT to UPT). Situation is illustrated by the Fig. 1.

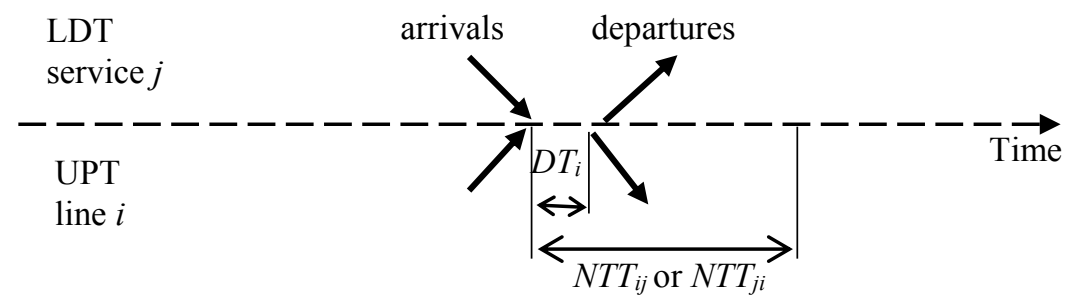

Fig. 1. Illustration of problem with $N T T_{i j}$ or $N T T_{j i}$

Presumption d) is a reason why it is not possible to realize so called 'timed transfer' [2]. It means that all services (vehicles) of LDT as well as UPT arrive short before defined moment. Mutual interchanges between all pairs of services (vehicles) are possible and then all the services will depart. Extension of dwell times $D T_{i}$ has limited effectivity, because of time loss caused for not-interchanging passengers.

Distribution of all LDT arrivals and departures all over the time can be the second reason next to it.

General point of view on situation is illustrated by the Fig. 2. The core of solution is to find time position of the first arrival of UPT line. This arrival will determine all other arrivals depended on line headway time $L H_{i}$. Departures are depended on dwell time $D T_{i}$ next to it. 


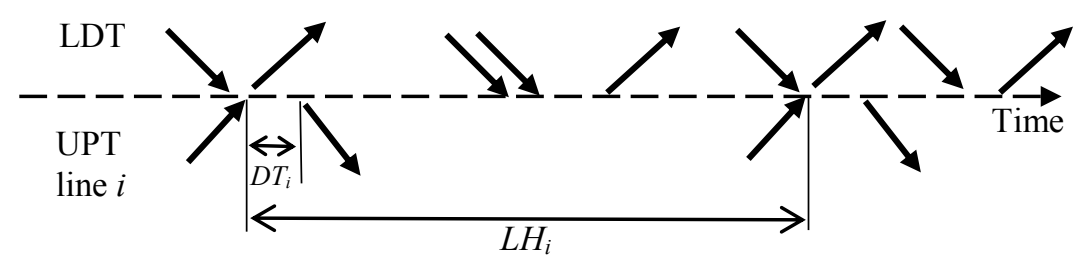

Fig. 2. Problem illustration - general point of view

\subsection{Literature overview}

Following paragraphs are elaborated as an analysis of state-of-art knowledge. Main factors influencing an interchange process are introduced as well.

Timetable must be realistic [3], robust and reliable. Robustness of 'timed transfer' synchronization is a theme of article [4]. There is a relation to quality. Reliability of operation is highlighted by the fact that the most of passengers use the same interchange node repeatedly. For example, $77 \%$ of passengers visit interchange node Moncloa (Madrid, Spain) more than 4 times per week [5].

Synchronization at interchange node, operational reliability, information availability and supplementary services belong to the set of 13 important passengers' evaluative criterions [6]. These 4 criterions can be related to interchange process.

There are many different approaches and criterions leading to solution of timetable synchronization problem. Basic overview of approaches published in time 1989-2015 is provided by [7]. Conclusion is that there is not one approach and one universal solution of this problem only. Individualized solution is necessary for each specific situation.

Time loss of interchanging passengers is estimated by [8]. The article is focused on interchange from backbone railway to supplementary bus lines in Beijing (China). The result is that time loss is linearly depended on supplementary line headway time $(L H)$.

There are mentioned two other important facts in the article [8] as well. Passengers consider time needed for interchange more sensitively in comparison with time spent on board of a vehicle. On the other hand, passengers often use time spent at interchange node for other activities (e.g. shopping) as well. Strict minimization of transfer time available for interchange is not unambiguous due to this fact.

Article [9] is focused on topological organization (spatial arrangement) of interchange node. Model is based on entropy. Synchronization of arrival and departure times is overshadowed.

Overcrowding of vehicles is discussed in article [10]. This can be negative effect influencing quality of public transport as well.

Complex overview of interchange nodes is provided by the European project NODES [11]. Research was lasting from 2012 to 2015. There is declared that utilization of transport models is very important. Application of models is presumed on 2 levels. Macroscopic level for models of entire transport systems as well as microscopic level for individual interchange nodes. Individualized passengers' point of view is emphasized. This project is mentioned also by [12] and by [13].

Article [14] is focused on technical features influencing time spent by waiting on underground stations. The main conclusions can be generalized and applied in other public transport subsystems as well. Number of doors in vehicle and size of horizontal and vertical gaps between platform and vehicle are identified as the main factors. Two factors can be added for UPT based on buses within discussion to this article. The first is number of vehicle doors used for boarding of passengers and the second is the way of passengers' check-in. This can have also serious influence on needed dwell (sojourn) time $D T_{i}$. 
Three different 'passenger types' are defined by [7]: transferring (interchanging), through and boarding passenger. This division is necessary for correct evaluation of time loss. A new type for alighting passengers should be added as the opposite option to group of boarding passengers for systematic evaluation.

Division of transport peaks into three types: double, triple and plain is proposed by [15]. It is an important fact, because extent of transport operation can vary in time. Solution of timetable synchronization problem must reflect it.

There are also articles focused on information systems providing on-line information about position of vehicles. Articles [16], [17] and [18] can be mentioned as examples.

The article [18] is also focused on bus bunching and on prevention of it. Bus bunching occurs, when vehicles are going in short subsequent headway time.

Linear programming model is presented by [19]. Simplex method is applied. Model is designed for timetable synchronization in multimodal hubs. It is dedicated to periodical transport services using different periods $(L H)$. There are two important facts. The first is decomposition of transfer time into parts. The second fact is that capacity of platforms and stops in hub is considered. This is an illustration that organization of transport operation is a complex problem with various conditions. Each of them can have an impact on final solution.

Mixed integer linear programming model is proposed for synchronization of last evening trains and buses by [2]. All interchange nodes served by one trunk (backbone) line must be synchronized together. Network approach is necessary in this case.

Mixed integer nonlinear programming model is presented by [20]. It is dedicated for optimization of timetable in underground networks. Target is to maximize number of synchronized interchanges in solved network. Two services are marked as 'synchronized' if value (length) of transfer time is in defined range. Benefit is that interchange times are not required to be minimized as most as possible. This effect can occur if a sum of transfer times would be minimized. Minimal interchange times can be a reason for amplification on unreliability in the case of delay. It is also declared by [20] that it is not possible to coordinate all the interchanges.

\subsection{Research hypothesis}

As it follows from problem statement and from analysis of state-of-art level of knowledge, interchange is one of critical parts of transport process. Reliability and attractiveness of public transport can be influenced. Maximal attention must be paid to organization of interchange process, including timetable synchronization. The effort of this article is to contribute to solution of these questions in the field of an interface between LDT and UPT.

Research hypothesis can be stated as: "There is an opportunity to synchronize UPT to LDT in the case of busy hub of LDT by respect to presumptions a) -d) mentioned on the beginning." There will be an effort to respect the most of effects mentioned within the analysis of state-of-art knowledge.

The aim of this article is to define and discuss mathematical optimization model supporting timetable synchronization of UPT and LDT services in busy hubs of LDT.

\section{Model formulation}

Researched task belongs to the scope of 'timetable synchronization problem'. In general, there are several ways how this problem can be solved [7]. Local conditions, specifics and requirements must be reflected. This chapter is focused on mathematical aspects of optimization and on formulation of the model in basic form. 
Primary, proposed optimization model is formulated as a nonlinear integer mathematical programming optimization model (MPOM) and implemented in the Solver of Microsoft Excel. Evolutionary algorithm of the Solver is applied. This model can be categorized as heuristic.

Secondary, an exhaustive-search algorithm (ESA) will be introduced as a parallel and extending way of solution. Algorithm of ESA is based on iterative setting of all possible values of independent variable(s) into the model. Target function value and other information are recorded for each iteration. Minimal recorded value of target function will determine the result (optimal solution). Naturally, computing time is depended on extent of solved task and on parameters of used computer.

Application of ESA can be helpful in two areas. The first is possibility to find optimal solution also in the case when computing capabilities for MPOM are exceeded. The second area is more important. Exhaustive searching provides evaluation (value of target function) for each individual way of solution (by each iteration of algorithm). These results can allow important extension of optimization possibilities. For instance, the effect of bus bunching [18] can be prevented by using of these data. Spare time position (e.g. the second best) could be used in this case.

Application of ESA next to MPOM deepens a difference to the models presented by $[2,19]$ and [20] as well. Technically, ESA is implemented as a macro in Microsoft Excel. This macro has been developed by using of Visual Basic for Application (VBA) programming language.

Both approaches to solution are based on the same mathematical expressions (1)-(10), respectively on the same model formulation.

\subsection{Model inputs}

There are 4 main groups of inputs necessary for optimization:

a) Timetable of all LDT services $j \in J$ expressed by all arrival times $L D T A_{j}$ and departure times $L D T D_{j}$ inserted in the form of a number of minute of a day (e.g. $0.00=0$ and $23.59=1439 \mathrm{~min}$ ).

b) Required line headways (intervals) $L H_{i}$ [min] and required dwell times $D T_{i}$ [min] for all UPT lines $i \in I$.

c) All transfer times $N T T_{i j}$ [min] needed for interchange from UPT line $i \in I$ to LDT service $j \in J$ and $N T T_{j i}$ [min] from LDT service $j \in J$ to the UPT line $I \in I$.

d) Origin-destination matrix $\mathbf{F}$ expressing intensities of passenger flows $f_{j i}$ and $f_{i j}$ [passengers/time] interchanging in all mutual pairs of LDT service $j \in J$ and UPT line $I \in I$ within solved time period $T_{p e r}$. This input is needed for the case of intensity approach to criterion only. See chapter 2.3 for details.

\subsection{Basic model variables}

There is one independent variable $x_{i}$ for each UPT line $i \in I$ only. Value of this variable determines whole timetable of the line. Value of $x_{i}$ is from interval $<0 ; L H_{i}$ ) and represents the first arrival to the hub in solved time period with the length of $T_{p e r}$ minutes.

All other arrivals and all departures of services on UPT line $i \in I$ are represented by dependent variables. Arrival time position of service coming on the $u$ position in order (in solved time period) $U P T A_{u}{ }^{i}$ can be found by the formula (1). It is valid that $U P T A_{1}{ }^{i}=x_{i}$ for the arrival of the first service in order.

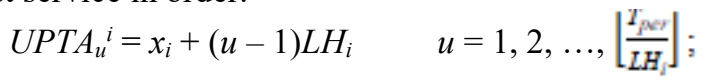


All departures $U P T D_{u}{ }^{i}$ of service $u$ on the UPT line $i$ can be found by using of dwell times $D T_{i}$, see formula (2).

$$
U P T D_{u}{ }^{i}=U P T A_{u}{ }^{i}+D T_{i} \quad u=1,2, \ldots,\left\lfloor\frac{x_{\text {ass }}}{L H_{j}}\right\rfloor ; \forall i \in I
$$

There are auxiliary variables applied in the model as well. These auxiliary variables support estimation of passengers' time loss. These variables will be mentioned in the chapter 2.3 focused on target functions (optimization criteria).

\subsection{Target function}

Decomposition of interchange process (transfer) is necessary for definition of target function. Applied decomposition is close to decompositions used by [5] and [19]. It is an evidence that it should be suitable for practical application. Decomposition is in the Fig. 3.

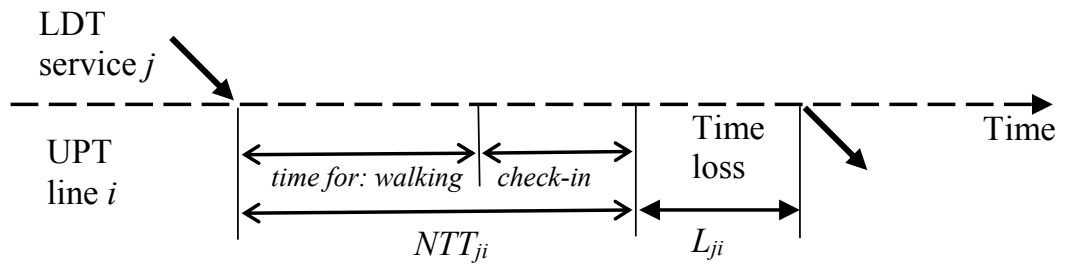

Fig. 3. Decomposition of Interchange Process Source: Author by using of [5, 19]

Passengers' time loss $L_{j i}$ [min] occurred by interchange from LDT service of $j \in J$ to UPT line $i \in I$ is crucial factor for construction of criteria to be optimized. Time loss is defined as a difference between value of time space (created by UPT departure and LDT arrival) and value of needed transfer time $N T T_{j i}[\mathrm{~min}]$. Analogic principle is valid also for interchange in reverse direction from UPT to LDT with passengers' time loss marked as $L_{i j}$ [min].

All the values of $N T T_{j i}$ or $N T T_{i j}$ must be defined with respect to walking possibilities as well as to time needed for check-in (e.g. purchasing of tickets). Transfer times $N T T_{j i}$ or $N T T_{i j}$ must reflect requirements of people with reduced mobility (PRM) as well. Time demands should be seriously increased due to necessity to use longer approaching routes suitable for wheelchairs, necessity to use lifts etc. For illustration, details to utilization of guiding lines for blind people can be found e.g. in article [21]. Check-in process is also limiting. In general, it is not possible to presuppose that all passengers will use all the possibilities to purchase tickets in advance although these possibilities are developing (especially the possibilities related to Internet). If check-in process is more complicated (e.g. equipped by security check) all features of this system must be reflected. Models of mass-serving systems theory should be useful in this case.

First, it is necessary to define 'ideal' values of UPT line $i \in I$ arrival (and departure) adequate for interchange from/to each individual LDT service $j \in J$ with no time loss ( $L_{j i}=0$ and $L_{i j}=0$ required). Comparison of these values with actually considered value of $x_{i}$ will allow work of optimization model.

Technically, it is better to do this by using of auxiliary variables. Ideal UPT arrival time

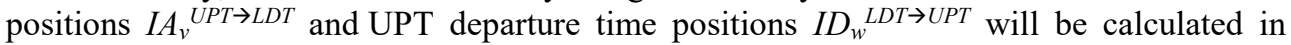
advance (tabulated) for each minute in solved time period $T_{p e r}$ with no regard if some LDT service is arriving or departing or not at that minute. These auxiliary variables express what will be an ideal solution when a LDT service will depart in the minute of $v$ or arrive in the minute of $w$. It is valid: $v \in \mathrm{N}_{0} \wedge 0 \leq v<T_{p e r}$ and $w \in \mathrm{N}_{0} \wedge 0 \leq w<T_{p e r}$. 
Formula (3) represents calculation of adequate arrival time $I A_{v}{ }^{U P T \rightarrow L D T}$ of UPT service for interchange to LDT service departing in minute of $v$.

$$
L A_{\mathrm{v}}^{U P T \rightarrow L D T}= \begin{cases}v-N T T_{\mathrm{if}} & \text { for } v \geq N T T_{\mathrm{if}} \\ v-N T T_{\mathrm{ij}}+T_{\text {per }} & \text { for } v<N T T_{\mathrm{if}}\end{cases}
$$

Formula (4) represents calculation of adequate departure time $I D_{w}{ }^{L D T \rightarrow U P T}$ of UPT service for interchange from LDT service arriving in minute of $w$.

$$
D_{W P}^{L D T \rightarrow U P T}= \begin{cases}w+N T T_{j p} & \text { for } T_{p e r}-1 \geq w+N T T_{j P} \\ w+N T T_{j P}-T_{p e r} & \text { for } T_{p e r}-1<w+N T T_{j P}\end{cases}
$$

It is possible to express (tabulate) passengers' time loss now. Formula (5) is used for passengers interchanging from UPT line $i$ to possible LDT service departing in minute of $v$. The first arrival of UPT line in solved time period with the length of $T_{p e r}$ is given by the value of $x_{i}$.

$$
L_{v}^{U P T \rightarrow L D T}= \begin{cases}\left(L A_{\mathrm{v}}^{U P T \rightarrow L D T} \bmod L H_{i}\right)-x_{i} & \text { for } x_{i} \leq\left(L A_{\mathrm{v}}^{U P T \rightarrow L D T} \bmod L H_{i}\right) \\ \left(L A_{\mathrm{v}}^{U P T \rightarrow L D T} \bmod L H_{i}\right)-x_{i}+L H_{i} & \text { for } x_{i}>\left(L A_{\mathrm{v}}^{U P T \rightarrow L D T} \bmod L H_{i}\right)\end{cases}
$$

Formula (6) is applied for passengers interchanging from LDT service arriving in minute of $w$ to UPT line $i$.

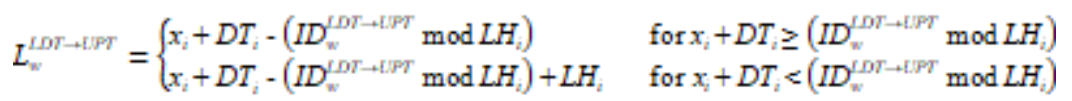

Passengers' time loss $L_{i j}$ or $L_{j i}$ occurring by mutual interchange between specific LDT service of $j \in J$ to UPT line of $i \in I$ is determined by using of pre-calculated (tabulated) adequate auxiliary variables $L_{v}{ }^{U P T} \rightarrow L D T$ and $L_{w}{ }^{L D T \rightarrow U P T}$ corresponding to actually estimated combination of LDT arrival (departure) of service $j \in J$ and the estimated value of $x_{i}$ for the UPT line of $i \in I$. Repeated calculation of time loss is not necessary by using of these auxiliary variables, but it is not excluded if needed.

Finally, target function can be formulated now. There are 2 parallel variants of criterion (target function) proposed. The first one is named as 'intensity approach' and the second one as 'uniform approach'.

'Actual' total time loss of all passengers ITL [min] is a criterion in the case of intensity approach. Passengers' time loss $L_{i j}$ or $L_{j i}$ is multiplied by number (flow intensity) of interchanging passengers $f_{i j}$ or $f_{j i}$ and summed. Target function in the case of intensity approach is expressed by formula (7).

To minimize

$$
\mathrm{ITL}=\sum_{i \in I} \sum_{j \in J}\left(f_{i j}-L_{i j}+f_{j i}-L_{j i}\right)
$$

There is necessary to have origin-destination matrix of flows of interchanging passengers $\mathbf{F}$ at disposal in the case of intensity approach. This should be a disadvantage of this approach, because transport survey could be needed. Second important fact is that this approach can lead to situation when resulting transfer times will be systematically shortened on interchanges which are used by the most of passengers. It should be attractive, but problematic in the point of view of system stability as well. These most preferred interchange relations can be disconnected in the case of delay when the subsequent 
connection will not wait. Passengers travelling on the less utilized relations may feel possibly extended interchange time as a disadvantage.

Second possible approach is uniform approach. This criterion follows two main facts. The first is network character of public transport providing services for all passengers travelling almost from everywhere to everywhere. The second is reduction of extent of input data, because origin-destination matrix $\mathbf{F}$ (transport survey) is not necessary. Calculation of total time loss reached by uniform approach UTL [min] is represented by formula (8).

To minimize

$$
\mathrm{UTL}=\sum_{i \in I} \sum_{j \in J}\left(L_{i j}+L_{j i}\right)
$$

All interchange relations (pairs of UPT and LDT services) are considered on the same level of importance like 1 passenger will interchange there. Problem could be that some interchange relations with low (or no) utilization are considered as well. This can have a negative impact on result as well. On the other hand, no relation will be preferred or notpreferred.

\subsection{Model constraints}

The variable $x_{i}$ represents departure time of the first service journey on optimized UPT line in time period for solution as it was stated. Formula (9) represents a constraint that the departure time $x_{i}$ will be located between the value of 0 and value of headway time on that UPT line. The value of 0 is permissible, the value of $L H_{i}$ not.

$$
0 \leq x_{i}<L H_{i}
$$

Formula (10) represents a formal constraint. The values of $x_{i}$ variable can be integer only (from a set of natural numbers). The value of 0 is also possible to be occurred.

$$
x_{i} \in N \cup\{0\}
$$

It is not necessary to determine any other formal constraints. There are not any other independent variables in the model. Formulas (9) and (10) ensure using of permissible values by all other dependent variables as well.

\section{Model in transport technology point of view}

This chapter is dedicated to important technological aspects of model implementation. In simple, some facts how to apply the model are mentioned. Some basic technological modifications of proposed model are introduced as well, but in short and with reduced emphasis on mathematical background.

\subsection{Considered time period}

There are different types of transport demand according to [15]. In general, extent of transport can vary in time according to changing transport demand and to other operational conditions. It means that there are different numbers of LDT services operated in the hub as well as possibly different line headways of UPT line $L H_{i}$ in different periods of time. It is necessary to divide time according to variations of transport extent (e.g. according to days working, Saturdays, Sundays or according to part of a day - peak, off-peak, morning, evening, night time periods etc.). Then it is possible to make this optimization according to 
each individually considered time period with different operational features. This is recommended procedure.

It is not necessary that the length of decomposed (considered) time period must be equal to the length of the (solved) time period $T_{p e r}$ used for optimization in the model. It is possible to make a projection of all LDT services to solved time period (e.g. $T_{p e r}=60 \mathrm{~min}$ ) after simple modification of model. Weights reflecting number of services operated at the same time can be add for distinction. For example, if services (e.g. trains) will arrive periodically at 55th minute of each hour during all day, 1 arrival will be assigned to the minute of 55 with the weight of 24 (number of repetitions in a day). This is valid for the case of $T_{p e r}=60 \mathrm{~min}$. Season coefficients are also at disposal, e.g. if a service is not operated during summer holidays it should be weighted as $10 / 12=0.83$ service (service is operated in 10 months from 12 in a year).

\subsection{Setting of transfer times}

Transfer times $N T T_{i j}$ and $N T T_{j i}$ set in an adequate way are crucial to meet passengers' needs. Too long values can cause passengers' time loss, too short can decrease the level of comfort. Interchange might to be inconvenient in both cases. Idea that passengers consider time spent by interchange more sensitively than time spent on board [8] must be pointed out once again in this case.

The aim of this modification is to point out that transfer times $N T T_{i j}$ and $N T T_{j i}$ can be defined for each UPT line individually. Shorter transfer times can be applied in the case of UPT line servicing such industrial area where the most of passengers are daily-commuters. These passengers usually do not purchase tickets every day. They usually know very well where to go and time demands for interchange can be reduced. On the other hand, extended transfer times $N T T_{i j}$ and $N T T_{j i}$ can be applied by a UPT line servicing such place significant for tourists. Tourists can search for information longer (possible language barrier, unfamiliarity with local specifics etc.), they can carry number of pieces of luggage etc. This can increase time demands in comparison with daily-commuters. Individual consideration of passengers' structure by each UPT line could be helpful.

Similar modifications of transfer times $N T T_{i j}$ and $N T T_{j i}$ can be related to a type of LDT service $j$ as well.

\subsection{Exclusion of some of LDT services from optimization}

This possibility is related to structure of passengers mentioned in the chapter 3.2 as well. Not all LDT services are attractive for all the passengers in the same way. For example, such international long-distance train is invited to be more attractive for tourists than for daily-commuters travelling by regional trains to the surroundings of the city. It is possible to select which LDT services can be excluded from optimization, but it can be done when the LDT service is quite unattractive. On the other hand, this can allow more convenient synchronization with LDT services which are effective (e.g. between UPT line servicing touristic objects and long-distance trains).

\subsection{Timetable synchronization within UPT subsystem based on data reached by ESA}

Finally, there is one important question more. In an extreme case when all the inputs will be equal (the same) in the case of all UPT lines, MPOM algorithm will find the same (equal) result for all the UPT lines. This will directly lead to bus bunching effect [18]. For example, all of lines operated in the same headway of $L H=20 \mathrm{~min}$ will depart e.g. in the moments of 
X.00, X.20 a X.40. All vehicles will drive together in a collective segment of route (e.g. from railway station to city centre). This is not suitable in transport technology point of view.

Parallel model ESA calculates values of target function ITL or UTL for all possible integer values of $x_{i} \in<0 ; L H_{i}$ ). In spite of the fact that the 'spare' values will be not optimal, 'spare' values of xi' can be used e.g. for finding of other time schedule creating more convenient timetable in collective segments of route. For example, UPT services can be ideally operated every $10 \mathrm{~min}$ in the case of 2 lines by $L H=20 \mathrm{~min}$ in collective segment of route. On the other hand, effectiveness of all timetable variants can be considered and such suitable compromise solution selected. Other conditions occurred in the UPT system (e.g. ensuring of interconnections of individual UPT lines at other nodes of UPT network) can be regarded as well.

\section{Case study: UPT Pardubice at the Pardubice main railway station}

City of Pardubice is located in the Czech Republic. It is a regional centre with about 90000 inhabitants. Illustration case study is made for Pardubice main railway station as an interchange node of railway transport (as LDT) and UPT.

Case study is based on timetable for 2016 [22]. Differences in extent of transport operation in 2016 and 2018 are not very important for the study to be reworked, because it is used for illustration purposes only.

There were registered 288 train arrivals and 287 train departures per day. Total number of bus or trolleybus UPT lines operated there was 19. The most of them are operated in both directions of drive.

UPT stop timetables are usually displayed by departures. Values of departures $y_{i}$ will be used for interpretation of results due to this. Relation between these variables is: $y_{i}=x_{i}+D T_{i}$. Arrivals $x_{i}$ were used within calculation.

Different needed transfer times $N T T_{i j}$ are used for interchanges from UPT to trains of different type. Following values are applied: $N T T_{i j}=4 \mathrm{~min}$ in the case of interchange to regional trains, $N T T_{i j}=6 \mathrm{~min}$ in the case of fast trains and $N T T_{i j}=8 \mathrm{~min}$ in the case of international or express trains (e.g. EuroCity, InterCity). It is due to the structure of passengers (see chapter 3.2). The value of $N T T_{j i}=5 \mathrm{~min}$ is applied for all interchanges from trains to UPT. Dwell time of UPT lines is set to the value of $D T_{i}=1 \mathrm{~min}$.

There are presented examples of 2 different UPT lines. The first is backbone trolleybus line No. 2. The second example deals with a supplementary bus line No. 26.

Uniform approach is applied, because OD matrix $\mathbf{F}$ is not at disposal. On the other hand, it is necessary to ensure 'systemic accessibility' for passengers travelling in all directions by this UPT system.

\subsection{Line No. 2, direction city centre}

This line is used for travelling to/from railway station as well as for travelling within city area. Housing estate Polabiny, main railway station, city centre and hospital are served by this line. All trains are taken into optimization (no one is excluded). There are 3 significant time periods created (see chapter 3.1 and Tab. 1) selected according to different line headway time. The values are: $L H_{2}=15 \mathrm{~min}$ valid for working days; $L H_{2}=20 \mathrm{~min}$ for weekends and $\mathrm{LH}_{2}=30 \mathrm{~min}$ for evening time (of all weekdays). 
Table 1. Optimization results - line No. 2, direction city centre

\begin{tabular}{|c|c|c|c|c|c|}
\hline \multirow{2}{*}{$\begin{array}{l}\text { Time } \\
\text { period }\end{array}$} & \multicolumn{2}{|c|}{ actual scheduled timetable 2016} & \multicolumn{2}{|c|}{ optimized (proposed) timetable } & \multirow{2}{*}{$\begin{array}{r}\text { Savings } \\
\text { of time } \\
\text { loss }\end{array}$} \\
\hline & $\begin{array}{r}\text { timetable } \\
y_{2}\end{array}$ & $\begin{array}{l}\text { total time loss } \\
\text { UTL [min] }\end{array}$ & $\begin{array}{r}\text { timetable } \\
y_{2}\end{array}$ & $\begin{array}{l}\text { total time loss } \\
\text { UTL [min] }\end{array}$ & \\
\hline $\begin{array}{c}\text { Working } \\
\text { day }\end{array}$ & $y_{2}=7,(22,37,52)$ & 17112 & $y_{2}=2,(17,32,47)$ & 13977 & $18.32 \%$ \\
\hline Weekend & $y_{2}=15,(35,55)$ & 7400 & $y_{2}=8,(28$ and 48$)$ & 6613 & $10.64 \%$ \\
\hline Evening & $y_{2}=2,(32)$ & 6814 & $y_{2}=23,(53)$ & 5761 & $15.45 \%$ \\
\hline
\end{tabular}

The ESA model can evaluate all possible solutions by total time loss UTL. Results are expressed according to all integer departure times of the first UPT service journey $x_{2} \in<0$; 15) min. The results are in the Fig. 4. The variants (sets of departures): $y_{2}{ }^{\prime}=11,(26,41,56)$ and $y_{2}{ }^{\prime \prime}=12,(27,42,57)$ should be relative good spare solutions when operational reasons do not allow to realize optimized solution, e.g. due to bus bunching.

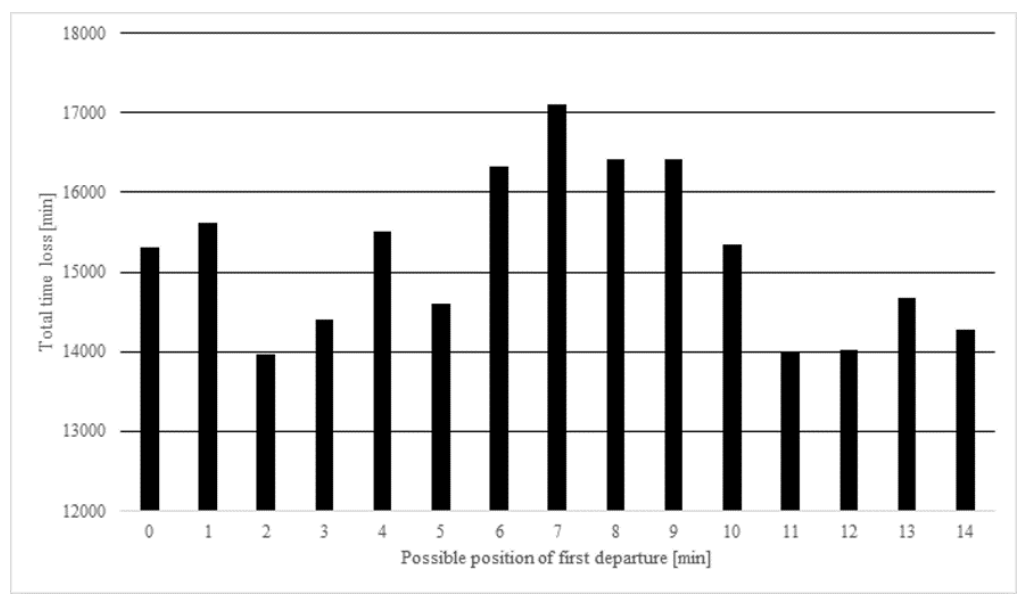

Fig. 4. Results of ESA - line No. 2 direction city centre (working day, all trains)

\subsection{Line No. 26}

This UPT line connects housing estate Dubina with the main railway station in a direct way (out of city centre). It is such kind of supplementary express line, operated by headway time of $L_{26}=60 \mathrm{~min}$ on working days only. Interchanging from/to long-distance trains is presupposed as dominant by this line. Regional railway transport is excluded from consideration for that reason (see chapter 3.3).

Optimized time arrival position of buses from Dubina to railway station is $x_{26}=50 \mathrm{~min}$ in each hour. Related total time loss is $U T L=11687 \mathrm{~min}$. Total time loss was $U T L=15152 \mathrm{~min}$ in reality. Applied arrival times were $x_{26}=38 \mathrm{~min}$ in forenoon and $x_{26}=51 \mathrm{~min}$ in afternoon time period. Total time loss could be reduced by $22.87 \%$ by 
MPOM or ESA models. Relative high values of time loss are caused by long headway time on the UPT line.

Optimized value for departures (in direction Dubina) is $y_{26}=5 \mathrm{~min}$ in each hour (time loss of $U T L=11477 \mathrm{~min})$. Applied departures were $y_{26}=24 \mathrm{~min}$ in forenoon and $y_{26}=11 \mathrm{~min}$ in afternoon time (related total time loss $U T L=15145 \mathrm{~min}$ ). UTL could be reduced by $24.22 \%$.

Impact of excluding of selected trains from consideration can be presented by the Fig. 5 . All possible departure time positions are evaluated by UTL using ESA in the Fig. 5 for the case when all the trains will represent prospective connection to the UPT line 26 (optimized departure $y_{26}=32 \mathrm{~min}$ ).

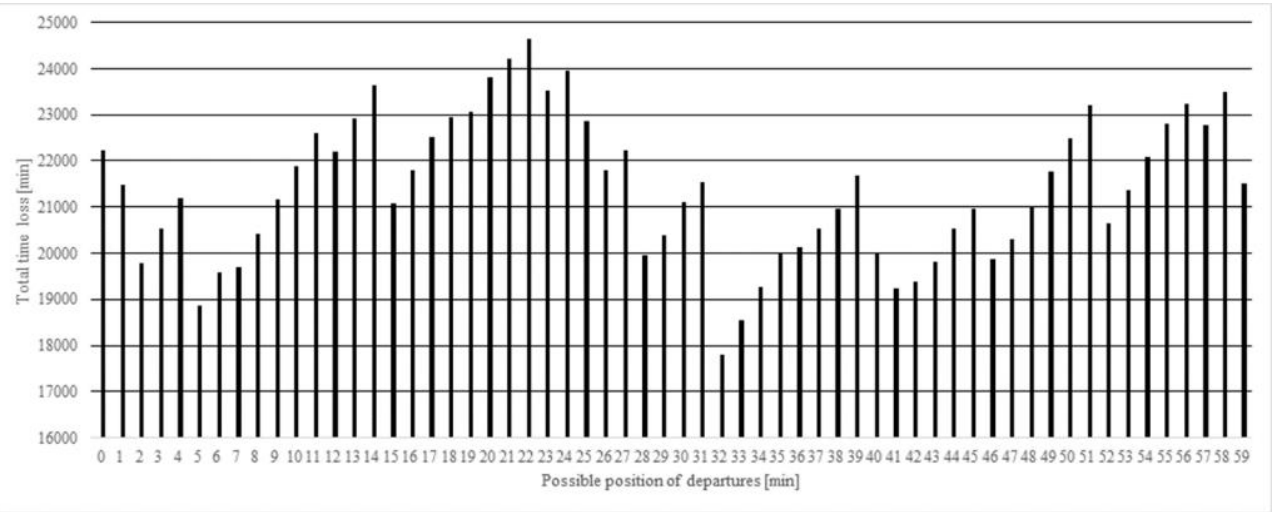

Fig. 5. Results of ESA - line No. 26 direction Dubina (working day, all trains)

Variability of utilization and technological possibilities of proposed model should be illustrated by this chapter 4.2 .

\section{Discussion}

Research hypothesis: "There is an opportunity to synchronize UPT to LDT in the case of busy hub of LDT by respect to presumptions a) - d) mentioned in the chapter 1 " is not possible to be rejected. The model creating scope of this article is an evidence. Documented reductions of total time loss UTL (by $10.64-24.22 \%$ in the case study) seem as significant and helpful.

It is documented that timetable synchronization is a complex problem influenced by a lot of other conditions, including results of optimizations in other fields. It is necessary to apply an individualized solution. Technological modifications mentioned in the chapter 4 allow this. Useful can be also application of ESA algorithm providing evaluation of all possible variants of UPT timetable as well. This can allow reaction on other conditions occurred in practice (like e.g. bus bunching protection).

All reasons and aspects of any technological modification must be clearly declared and discussed (defended) when needed. Possible modification can totally change the meaning of the result.

Mathematical and software implementation is relative simply (Microsoft Excel).

Proposed model should be categorized as supporting. There is an effort to create possibilities how to reflect other technological aspects of transport operation. Exhaustively searched results and subsequent evaluation of them could help to fulfil this presumption. 
Model could be helpful for UPT systems using longer line headways $L H_{i}$, because time spent for waiting can be significant. On the other hand, model can be applied by all integer positive values of $L H_{i}$.

Interesting question is also if uniform (formula 8) or intensity (formula 7) approach to criterion should be applied. Uniform approach is more convenient due to reduced volume of needed data, but also due to systemic approach to solution. The most utilized interchanges are not preferred like in intensity approach. Transfer time will be shortened in the case of very used interchanges and intensity approach. This advantage could be a problem in the point of view of reliability in the case of delay. Interchanges with shorter transfer time are more sensitive (possible time reserve is reduced).

\section{Conclusions}

The research aim of this article is fulfilled. Optimization model and two parallel ways MPOM and ESA of solution are proposed. Reduction of total time loss in reached solutions could be significant (as it is documented by presented case study). Model belongs to analytical tools providing data able to be helpful in overall transport planning process.

It is declared that not only one single way of solution is at disposal there. Some model modifications based on technological effects are presented. Active and sensible approach to model modifications it can lead to more adequate results of optimization.

There are also possibilities how to extend this research. Research how to use data provided by ESA can be one of possible extensions. Models for synchronization of UPT lines in collective sections of a route can be an example of possible development of proposed solution.

\section{References}

1. J. Bulíček Integrace systémů osobni dopravy - technologie provozu přestupnich uzli̊. University of Pardubice, habilitation thesis, in Czech (2015). http://hdl.handle.net/10195/66917

2. X. Dou, X. Meng, X. Guo Bus schedule coordination for the last train service in an intermodal bus-and-train transport network. Transport. Res. Part C, 60 360-376 (2015), http://dx.doi.org/10.1016/j.trc.2015.09.006

3. T.P. Hutchinson Transportation Planning and Technology, 34:8 747-758 (2011), http://dx.doi.org/10.1080/03081060.2011.613584

4. L.M. Kieu, et al. An investigation of timed transfer coordination using event-based multi agent simulation. Transport. Res. Part C (2017). http://dx.doi.org/10.1016/j.trc.2017.02.018

5. D. Lois, A. Monzón, S. Hernandéz Analysis of satisfaction factors at urban transport interchanges: Measuring travellers' attitudes to information, security and waiting. Transport Policy (2017) http://dx.doi.org/10.1016/j.tranpol.2017.04.004

6. J. Kleprlík, J. Matuška The demand for public transport and modeling decision-making process of passengers. Proceedings of $\mathbf{2 1}^{\text {st }}$ Int. Scientific Conf.Transport Means 2017 (2017), 20-22. September 2017, Kaunas, Lithuania, p.197-202, ISSN 1822-296X.

7. Y. Wu, J. Tang, Y. Yu, Z. Pan 2015. A stochastic optimization model for transit network timetable design to mitigate the randomness of travelling time by adding slack time. Transport. Res. Part C, 52 15-31 (2015) http://dx.doi.org./10.1016/j.trc.2014.12.012

8. S. Guo, L. Yu, X. Chen, Y. Zhang. Modelling waiting time for passengers transferring from rail to buses, Transportation Planning and Technology, 34:8 795-809 (2011), http://dx.doi.org/10.1080/03081060.2011.613589

9. L. Sun, J. Rong, L. Yao, H. Xu, H. Liu. Entropy-based estimation of transfers in a terminal, $\begin{array}{lllll}\text { Transportation Planning and Technology, 35:3 303-315 } & \text { (2012) }\end{array}$ http://dx.doi.org/10.1080/03081060.2012.671038 
10. V. Trozzi, I. Kaparias, M.G.H.Bell, G. Gentile A dynamic route choice model for public transport networks with boarding queues, Transportation Planning and Technology, 36:1 44-61 (2013) http://dx.doi.org/10.1080/03081060.2012.745720

11. NODES - EU project websites. Available from Internet: http://www.nodes-interchanges.eu

12. J. Carpio-Pinedo, J.A. Martínez-Conde, F.L. Daudén. Procedia - Social and Behavioral Sciences, 160 (2014), 224-233. XI Congreso de Ingenieria del Transporte (CIT 2014). http://dx.doi.org/10.1016/j.sbspro.2014.12.134

13. L. Lucietti, C. Hoogendoorn, I. Cré Transportation Research Procedia, 14 1240-1249 (2016) http://dx.doi.org/10.1016/j.trpro.2016.05.195

14. X. Karekla, N. Tyler Transportation Planning and Technology, 35:5 525-543 (2012) http://dx.doi.org/10.1080/03081060.2012.693267

15. X. Jiang, X. Guo Procedia - Social and Behavioral Sciences, 138 (2014), 501-509. http://dx.doi.org/10.1016/j.sbspro.2014.07.229

16. X. Song, J. Teng, G. Chen, Q. Shu Procedia - Social and Behavioral Sciences, 96 (2013), 22872299. http://dx.doi.org/10.1016/j.sbspro.2013.08.258

17. J. Argote-Cabanero, C.F. Daganzo, J.W. Lynn Res. Part B, 81 (2015), 146-160. http://dx.doi.org/10.1016/j.trb.2015.09.003

18. S. Rashidi, P. Ranjitkar, O. Csaba, A. Hooper Transportation Research Procedia, 25C (2017), 1444-1456. http://dx.doi.org/10.1016/j.trpro.2017.05.170

19. F. Kaakai, S. Hayat, A. El Moundi, S. Kapoor Schedule coordination for a bimodal connection chain into a Multimodal Hub. 11 ${ }^{\text {th }}$ IFAC Symposium on Control in Transportation Systems, Delft, August 29-30-31, (2006), 525-530.

20. X. Guo, H. Sun, J. Wu, J. Jin, J. Zhou, Z. Gao Multiperiod-based timetable optimization for metro transit networks. Transport. Res. Part B, 96 (2017), 46-67. http://dx.doi.org./10.1016/j.trb.2016.11.005

21. J. Koštálová, J. Matuška Save guiding of visually impaired people - artificial guiding lines arrangements in the infrastructure. In Proceeding of International Conferences on Traffic and Transport Engineering, Belgrade, 24. - 25. 11. (2016). p. 1073-1080. ISBN 978-86-916153-3-8 http://hdl.handle.net/10195/67378

22. IDOS timetable websites. CHAPS and MAFRA. Available from Internet: http://jizdnirady.idnes.cz/ 